\title{
Efeito de probiótico no desenvolvimento de leitões desafiados com Escherichia coli
}

\author{
Effect of probiotic on the development of piglets challenged with Escherichia coli
}

\section{Edison de ALMEIDA ${ }^{1}$; Simone Maria Massami Kitamura MARTINS ${ }^{1}$; Abrão Ferreira Antonio ABRAHÃO'; André Furugen Cesar de ANDRADE²; Andrea Micke MORENO³; Larissa José PARAZZI ${ }^{1}$; Tácia Antunes Del SANTO ${ }^{1}$; Aníbal Sant ${ }^{\prime A n n a ~ M O R E T T I ~}{ }^{1}$}

\author{
${ }^{1}$ Laboratório de Pesquisa em Suínos do Departamento de Nutrição e Produção Animal da Faculdade de Medicina Veterinária e \\ Zootecnia da Universidade de São Paulo, Pirassununga - SP \\ ${ }^{2}$ Departamento de Reprodução Animal da Faculdade de Medicina Veterinária e Zootecnia da Universidade de São Paulo, São \\ Paulo - SP \\ ${ }^{3}$ Laboratório de Sanidade Suína do Departamento de Medicina Veterinária Preventiva e Saúde Animal da Faculdade de Medicina \\ Veterinária e Zootecnia da Universidade de São Paulo, São Paulo-SP
}

\begin{abstract}
Resumo
Foram utilizados 72 animais aos 21 dias de idade para averiguar o efeito do probiótico no desempenho de leitões desafiados com E. coli. O delineamento foi em blocos casualizados, com arranjo fatorial $2 \times 2$. Os quatro tratamentos foram: probiótico com desafio; probiótico sem desafio; sem probiótico com desafio; sem probiótico e sem desafio. O probiótico Protexin $^{\mathrm{Tm}}$ foi administrado via ração, dos 21 aos 63 dias de idade e aos 35 dias, os animais dos tratamentos 1 e 3 foram desafiados. As variáveis analisadas foram: peso médio aos 35, 49 e 63 dias de idade; ganho diário de peso dos 35 aos 49 dias; dos 49 aos 63 dias; dos 35 aos 63 dias e escore fecal. As variáveis ganho diário de peso, peso médio e escore fecal foram submetidos à análise de variância pelo PROC GLM. Em relação ao peso e ganho de peso verificou-se ação benéfica do probiótico quando se associaram estas características com a ocorrência de diarréia pós-desafio e a redução da E. coli. Concluiu-se que o probiótico no modelo experimental aplicado apresentou efeito notório nas reduções de $E$. coli e da diarréia, podendo exercer influência no desempenho dos leitões.
\end{abstract}

Palavras-chave: Suíno. Desempenho. Diarréia. Contagem de E. coli nas fezes.

\begin{abstract}
We used 72 piglets at 21 days old and the aim was to determine the effect of probiotics on performance of piglets challenged with E. coli. The design was a randomized block design with $2 \times 2$ factorial arrangement. The four treatments were: with probiotic and challenge, with probiotic without challenge, without probiotic with challenge, without probiotic and challenge. The probiotic was administered via Protexin ${ }^{\mathrm{T}}$ diet, from 21 to 63 days old and 35 days, the animals in treatments 1 and 3 were challenged. The variables were: average weight at 35, 49 and 63 days old, daily weight gain from 35 to 49 days, 49 to 63 days, from 35 to 63 days and fecal score. The variables average weight, daily weight gain and diarrhea score were subjected to analysis of variance by PROC GLM. In terms of weight and weight gain was found beneficial effect of probiotic when these characteristics were associated with the occurrence of diarrhea post-challenge and the reduction of E. coli. It was concluded that the probiotic used in the experimental model showed clear effect on the reductions of E. coli and diarrhea, are relatively influenced in the performance of piglets.
\end{abstract}

Keywords: Swine. Performance. Diarrhea. Faecal E. coli quantification.

\section{Introdução}

Nos últimos anos, o controle das doenças entéricas nos leitões tem sido feito através do uso generalizado de antibióticos em níveis terapêuticos e sub-terapêuticos ${ }^{1}$. No entanto, os antibióticos comumente utilizados como promotores de crescimento começaram a ser questionados por reduzirem a população de organismos benéficos à saúde e integridade intestinal, aliado a resistência bacteriana cruzada ${ }^{2}$.
Esse fato tem gerado maior interesse na utilização

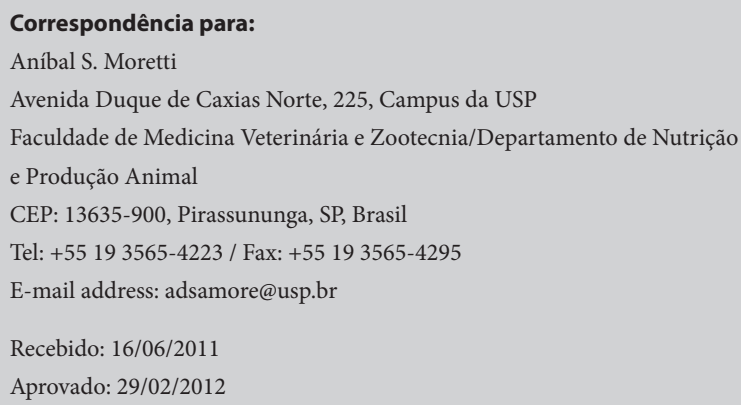


de fontes alternativas aos antibióticos que funcionem como promotores de crescimento o que motivou a indústria farmacêutica a fabricar novos produtos que seriam utilizados em substituição³.

A modificação da microbiota através da dieta pode ser uma alternativa para amenizar o estresse pósdesmama ${ }^{4}$. A microbiota desejada é obtida através da utilização dos probióticos, prebióticos e/ou simbióticos ${ }^{5}$.

Os probióticos, considerados alimentos funcionais, geralmente são empregados nas fases iniciais de vida dos leitões e antecedendo períodos críticos ${ }^{6}$. Esses microrganismos podem atuar por exclusão competitiva e antagonismo direto ${ }^{7}$, estímulo ao sistema imunológico $^{8}$, efeito nutricional ${ }^{9}$ e supressão da produção de amônia e neutralização de enterotoxinas ${ }^{10}$.

Diversos estudos têm demonstrado os efeitos dos probióticos, havendo melhora no ganho de peso e conversão alimentar ${ }^{11}$, na absorção de nutrientes pela produção de enzimas, vitaminas e síntese de aminoácidos essenciais ${ }^{12}$. Além disso, tem sido sugerido que a utilização de probióticos pode diminuir as contagens de microrganismos patogênicos no trato gastrointestinal ${ }^{13}$. Os probióticos mais comumente utilizados são as cepas de origem entérica, que pertencem ao grupo vulgarmente conhecido como bactérias produtoras de ácido láctico Lactobacillus sp, Bifidobacterium sp. e Streptococcus sp. ${ }^{14}$.

$\mathrm{Na}$ evolução dos estudos com probióticos e fitocompostos verificou-se a necessidade de introduzir o desafio programado para melhor aferir a ação dos agentes benéficos. Esse tipo de desafio também foi relatado por Van Dijk et al. ${ }^{15}$ sendo a E. coli a mais utilizada em estudo da diarréia pós-desmama, embora haja outras bactérias que podem ser empregadas nos desafios, tais como as cepas de S. typhimurium do tipo T8907 Sig$\mathrm{ma}^{16}$ e do tipo LSS $90 / 05^{17}$.

O objetivo do estudo foi averiguar a influência do probiótico Protexin ${ }^{\mathrm{Tn}}$ no desempenho de leitões desafiados com Escherichia coli.

\section{Material e Método}

O experimento foi realizado no Laboratório de Pesquisa em Suínos (LPS) da FMVZ-USP. A creche possuía gaiolas de alvenaria suspensas com piso aquecido e parcialmente ripado medindo aproximadamente 0,6 $\mathrm{m}^{2}$ constituídas de comedouros semi-automáticos e bebedouros tipo chupeta. Utilizaram-se 72 leitões híbridos aos 21 dias de idade e peso médio de $6,5 \mathrm{~kg}$, distribuídos em 24 gaiolas (três animais por gaiola), totalizando seis repetições por tratamento. O delineamento empregado foi em blocos casualizados, com arranjo fatorial $2 \times 2$, sendo um fator o uso ou não do probiótico Protexin ${ }^{\mathrm{mix}}$, composto por Bifidobacterium bifidum, Enterococcus faecium, Lactobacillus acidophilus, Lactobacillus bulgaricus, Lactobacillus plantarium, Lactobacillus rhamnosus e Streptococcus thermophilus e o outro fator o emprego ou não do desafio com cepa de E. coli positiva para as toxinas LT e STb e para as fímbrias K88 e F18.

Os animais foram distribuídos em quatro tratamentos: probiótico com desafio; probiótico sem desafio; sem probiótico com desafio; sem probiótico e sem desafio. O probiótico foi administrado via ração (100g/ ton), dos 21 aos 63 dias de idade. Aos 35 dias, trinta e seis animais foram inoculados com a cepa de E. coli (Amostra LSS 164/04), por via oral, na dose de $1 \mathrm{~mL}$ e na concentração de $1 \times 10^{8} \mathrm{UFC} / \mathrm{mL}$. Os leitões desafiados foram alojados em sala distinta, sendo isolado todo o manejo e os materiais utilizados. A água e a alimentação foram fornecidas ad libitum, seguindo as recomendações nutricionais (NRC) ${ }^{18}$.

O escore fecal foi observado $24 \mathrm{~h}$ antes do desafio, 24 e 48 horas e 9 dias após o desafio, e classificado em: 0 = fezes sólidas-normal 1 = fezes mais moles que o normal-diarréia leve; 2 = fezes cremosas-diarréia moderada e 3 = fezes líquidas-diarréia severa. Aos 35, 42 e 49 dias de idade foram coletadas amostras de fezes de 11 animais/tratamento para contagem de E. coli realizada no Laboratório de Sanidade Suína da FMVZ- 
USP. Utilizou-se um grama de fezes homogeneizada em $9 \mathrm{~mL}$ de solução salina estéril, sendo esta suspensão submetida a diluição seriada na razão de 1:10 até a diluição $1 \times 10^{-12}$. Um volume de $100 \mu \mathrm{L}$ de cada diluição foi semeado em agar McConkey e após 24 horas de incubação foi realizada a contagem de unidades formadoras de colônia.

Além disso, foi averiguado o peso aos 35, 49 e 63 dias de idade e os ganhos diário de peso compreendidos entre 35 a 49 dias (GDP49); 49 a 63 dias (GDP63) e 35 aos 63 dias (GDPTotal).

As variáveis ganho diário de peso, o peso, o escore fecal e a contagem de coliformes foram submetidos à análise de variância pelo PROC GLM (SAS) ${ }^{19}$. Os dados da contagem de coliformes fecais foram transformados em logaritmo $\left(\log _{10}\right)$ antes da análise de variância. As análises dos tratamentos foram realizadas pelo teste de Fisher (LSD), a 5\% de significância.

\section{Resultados}

\section{Desempenho dos leitões}

A tabela 1 apresenta os valores de GDP dos animais nos diferentes tratamentos. No período de 21 a 35 dias de idade, observou-se uma pequena variabilidade nos pesos sendo 6,$78 ; 6,66 ; 6,95$ e $6,23 \mathrm{~kg}$ para os grupos probiótico com desafio e sem desafio, sem probiótico com desafio e sem desafio, respectivamente. Essa variação no peso refletiu no GDP no mesmo período, sendo observado ganhos de 320; 330; 260 e 340g/dia para os animais dos tratamentos probiótico com desafio e sem desafio, sem probiótico com desafio e sem desafio, respectivamente.

No ganho de peso (GDP49) subseqüente verificouse interação $(\mathrm{P}<0,05)$ entre probiótico e E. coli. A diferença $(P<0,05)$ foi observada nos animais do tratamento sem probiótico com desafio em comparação ao probiótico com desafio e sem probiótico sem desafio (652 vs 555 e $557 \mathrm{~g} /$ dia, respectivamente). Os leitões do grupo probiótico sem desafio apresentaram ganho semelhante ao sem probiótico com desafio e este não diferiu dos demais tratamentos.

Dos 49 aos 63 dias de idade não foram detectadas diferenças $(\mathrm{P}>0,05)$. Houve alterações do ponto de vista numérico no padrão de GDP, sendo destacado o diferencial apresentado pelos animais do grupo sem probiótico com desafio. Enquanto que para os outros tratamentos tenha se observado um padrão positivo de crescimento, para o sem probiótico com desafio

Tabela 1 - Médias e erro padrão da média (SEM) dos ganhos diários de peso: GDP49 (35 a 49 dias), GDP63 (49 a 63 dias), GDPTotal (35 a 63 dias), em gramas/dia de leitões alimentados com dietas contendo probiótico e desafiados com E. coli

\begin{tabular}{|c|c|c|c|c|}
\hline \multicolumn{2}{|c|}{ Tratamento } & \multirow{2}{*}{ GDP49 } & \multirow{2}{*}{ GDP63 } & \multirow{2}{*}{ GDPTotal } \\
\hline Probiótico & E. coli & & & \\
\hline+ & + & $555^{\mathrm{b}}$ & 712 & 633 \\
\hline+ & - & $590^{\mathrm{ab}}$ & 732 & 662 \\
\hline- & + & $652^{\mathrm{a}}$ & 577 & 615 \\
\hline- & - & $557^{\mathrm{b}}$ & 697 & 627 \\
\hline \multicolumn{2}{|c|}{ SEM } & 0,01 & 0,03 & 0,01 \\
\hline \multicolumn{5}{|c|}{ Efeitos principais } \\
\hline \multicolumn{2}{|c|}{+ Probiótico } & - & 722 & 647 \\
\hline \multicolumn{2}{|c|}{ - Probiótico } & - & 637 & 621 \\
\hline \multicolumn{2}{|c|}{+ E. coli } & - & 644 & 624 \\
\hline \multicolumn{2}{|c|}{ - E. coli } & - & 714 & 644 \\
\hline \multicolumn{5}{|c|}{ Fonte de variação $(\mathrm{P})$} \\
\hline \multicolumn{2}{|c|}{ Probiótico } & 0,2191 & 0,1709 & 0,3710 \\
\hline \multicolumn{2}{|c|}{ E. coli } & 0,2435 & 0,2559 & 0,5004 \\
\hline \multicolumn{2}{|c|}{ Probiótico x E. coli } & 0,0170 & 0,4133 & 0,7778 \\
\hline
\end{tabular}


houve uma queda da qual não diferiu $(\mathrm{P}>0,05)$ entre os tratamentos.

Os pesos médios aos 35, 49 e 63 dias de idade não apresentaram interação $(\mathrm{P}>0,05)$ e, tampouco, efeito dos fatores separadamente. Ambos os tratamentos que receberam probiótico (com e sem desafio) tiveram os maiores pesos aos 35 dias, em comparação ao sem probiótico (com e sem desafio). Detectou-se tendência à interação $(\mathrm{P}=0,0609)$ para o peso aos 49 dias, não sendo verificado efeito $(P>0,05)$ para os fatores em separado. Em relação ao P63, foi observada uma tendência à significância $(\mathrm{P}=0,0641)$ para o fator desafio, comprovando a efetividade deste (Tabela 2).

\section{Escore Fecal}

Associativamente ao desempenho foi analisado o escore fecal, não sendo observado efeito de interação e, tampouco, dos fatores em separado 24 horas antes da inoculação com E. coli. Os animais dos tratamentos probiótico com desafio e sem probiótico com desafio apresentaram os maiores escores, 1,00 e 1,17, respectivamente, neste período, demonstrando uma diarréia leve (Tabela 3). Os animais do probiótico sem desafio e sem probiótico sem desafio apresentaram valores abaixo do escore 1, 0,50 e 0,83, respectivamente.
Constatou-se, 24 horas após o desafio, significância dos fatores em separado, mas não a interação entre os mesmos. Os leitões do grupo probiótico com desafio manteve a mesma resposta (escore 1,00 ), sendo que o sem probiótico com desafio apresentou um aumento de escore de 1,17 para 1,67, tendendo para uma diarréia moderada. Já os animais do probiótico sem desafio mantiveram as fezes normais com escore abaixo de $1(0,50)$, enquanto no sem probiótico sem desafio verificou-se uma variação no escore de 0,83 para 1,00 chegando a apresentar uma diarréia leve.

Ao avaliar os animais 48 horas após a inoculação, detectou-se novamente efeito significativo para os fatores isoladamente, não havendo interação. Foi verificado que o probiótico com e sem desafio e sem probiótico com desafio apresentaram resultados semelhantes ao período anterior, havendo diferença somente nos animais sem probiótico sem desafio, que modificou o escore de 1,00 para 0,83 demonstrando uma melhora na consistência das fezes.

Em relação ao $9^{\circ}$ dia após o desafio foi averiguado uma interação significativa entre os fatores probiótico e desafio $(\mathrm{P}=0,0209)$, sendo apresentados os menores escores os animais dos tratamentos sem probió-

Tabela 2 - Médias e erro padrão da média (SEM) dos pesos médios aos 35 dias de idade, peso aos 49 dias e peso aos 63 dias, nos tratamentos, em quilogramas de leitões alimentados com dietas contendo probiótico e desafiados com E. coli

\begin{tabular}{|c|c|c|c|c|}
\hline \multicolumn{2}{|c|}{ Tratamento } & \multirow{2}{*}{ P35 } & \multirow{2}{*}{ P49 } & \multirow{2}{*}{ P63 } \\
\hline Probiótico & E. coli & & & \\
\hline+ & + & 11,22 & 18,97 & 28,91 \\
\hline+ & - & 11,32 & 19,57 & 29,81 \\
\hline- & + & 10,59 & 19,73 & 27,82 \\
\hline- & - & 11,02 & 18,80 & 28,57 \\
\hline \multicolumn{2}{|c|}{ SEM } & 0,15 & 0,20 & 0,38 \\
\hline \multicolumn{5}{|c|}{ Efeitos principais } \\
\hline \multicolumn{2}{|c|}{ + Probiótico } & 11,27 & 19,27 & 29,37 \\
\hline \multicolumn{2}{|c|}{ - Probiótico } & 10,81 & 19,27 & 29,19 \\
\hline \multicolumn{2}{|c|}{+ E. coli } & 10,90 & 19,35 & 28,74 \\
\hline \multicolumn{2}{|c|}{ - E. coli } & 11,17 & 19,19 & 29,72 \\
\hline \multicolumn{5}{|c|}{ Fonte de variação $(\mathrm{P})$} \\
\hline \multicolumn{2}{|c|}{ Probiótico } & 0,1443 & 0,9880 & 0,8802 \\
\hline \multicolumn{2}{|c|}{ E. coli } & 0,3905 & 0,6797 & 0,0641 \\
\hline \multicolumn{2}{|c|}{ Probiótico x E. coli } & 0,5931 & 0,0609 & 0,5895 \\
\hline
\end{tabular}


Tabela 3 - Valores médios e erro padrão da média (SEM) do escore fecal de leitões alimentados com dietas contendo probiótico e desafiados com E. coli

\begin{tabular}{|c|c|c|c|c|c|}
\hline \multicolumn{2}{|c|}{ Tratamento } & \multirow{2}{*}{$\begin{array}{c}24 \mathrm{~h} \text { antes desafio } \\
\text { E. coli }\end{array}$} & \multirow{2}{*}{$\begin{array}{l}\text { 24h após desafio } \\
\text { E. coli }\end{array}$} & \multirow{2}{*}{$\begin{array}{c}\text { 48h após desafio } \\
\text { E. coli }\end{array}$} & \multirow{2}{*}{$\begin{array}{c}9 \text { dias após desafio } \\
\text { E. coli }\end{array}$} \\
\hline Probiótico & E. coli & & & & \\
\hline+ & + & 1,00 & 1,00 & 1,00 & $0,83^{\mathrm{ab}}$ \\
\hline+ & - & 0,50 & 0,50 & 0,50 & $0,67^{\mathrm{b}}$ \\
\hline- & + & 1,17 & 1,67 & 1,67 & $1,50^{\mathrm{a}}$ \\
\hline- & - & 0,83 & 1,00 & 0,83 & $0,17^{\mathrm{b}}$ \\
\hline \multicolumn{2}{|c|}{ SEM } & 0,14 & 0,11 & 0,13 & 0,15 \\
\hline \multicolumn{6}{|c|}{ Efeitos principais } \\
\hline \multicolumn{2}{|c|}{ + Probiótico } & 0,75 & 0,75 & 0,75 & - \\
\hline \multicolumn{2}{|c|}{ - Probiótico } & 1,00 & 1,33 & 1,25 & - \\
\hline \multicolumn{2}{|c|}{+ E. coli } & 1,08 & 1,33 & 1,33 & - \\
\hline \multicolumn{2}{|c|}{ - E. coli } & 0,67 & 0,75 & 0,67 & - \\
\hline \multicolumn{6}{|c|}{ Fonte de variação $(\mathrm{P})$} \\
\hline \multicolumn{2}{|c|}{ Probiótico } & 0,3765 & 0,0011 & 0,0323 & 0,7241 \\
\hline \multicolumn{2}{|c|}{ E. coli } & 0,1473 & 0,0011 & 0,0061 & 0,0043 \\
\hline \multicolumn{2}{|c|}{ Probiótico x E. coli } & 0,7661 & 0,5936 & 0,4521 & 0,0209 \\
\hline
\end{tabular}

0 = fezes normais; 1 = fezes mais moles que a normal - pastosas; 2 = diarréia moderada - cremosa e 3 = diarréia severa - líquida

tico sem desafio, probiótico sem desafio e probiótico com desafio $(0,17,0,67$ e 0,83 , respectivamente) em comparação aos sem probiótico com desafio com escore 1,50. Além disso, verificou-se que os leitões sem probiótico com desafio diferiram dos probiótico sem desafio e sem probiótico sem desafio (1,50 vs 0,67 e 0,17 , respectivamente), mas não diferiram do probiótico com desafio $(0,83)$. Este último tratamento não diferiu dos demais, apesar de ter havido uma evolução indicativa de manutenção do escore no período estudado.

\section{Contagem de coliformes}

Aos 35 dias verificou-se efeito $(\mathrm{P}<0,05)$ apenas do probiótico, tendo sido observados os dois maiores valores no tratamento sem probiótico, comparado aos tratamentos com probiótico 7,73 e 7,54 vs 6,82 e 6,09 $\log 10 \mathrm{UFC/g}$ fezes, para sem probiótico com e sem desafio $v s$ probiótico com e sem desafio, respectivamente, conforme descrito na tabela 4 . Aos 42 dias detectou-se efeito do desafio e foi possível observar comportamento diferenciado da bactéria, pois nos tratamentos com probiótico houve um aumento em 0,27 e $0,36 \log _{10}$ UFC/g fezes, para a presença ou ausência da bactéria; por outro lado, os tratamentos sem probiótico tiveram uma redução de 2,18 e 0,82 $\log _{10}$ UFC/g fezes, respectivamente.

Aos 14 dias após a inoculação foi verificado aumento de E. coli nas fezes em todos os tratamentos, porém a proporção dos que receberam o probiótico foi mantida em 0,64 e 0,18 $\log _{10}$ UFC/g fezes, com e sem desafio, respectivamente; já para os leitões sem probiótico o aumento foi praticamente proporcional à redução ocorrida dias antes: 2,64 e 1,0 $\log _{10} \mathrm{UFC} / \mathrm{g}$ fezes, com e sem desafio, respectivamente. Neste período também houve efeito $(\mathrm{P}<0,05)$ apenas para probiótico. Os animais dos tratamentos probiótico sem desafio e sem probiótico sem desafio não foram desafiados com $E$. coli, mas constatou-se a presença do agente; embora tenha se tomado as devidas precauções para evitar a contaminação, esse fato aconteceu, acredita-se que pela proximidade das salas.

Aos 49 dias de idade observou-se que os animais do grupo sem probiótico com desafio apresentaram maior eliminação de $E$. coli nas fezes $\left(8,00 \log _{10} \mathrm{UFC} / \mathrm{g}\right.$ fezes) em relação aos demais tratamentos; possivelmente este fato foi propiciado pela maior colonização que ocorreu no início do desafio. De alguma forma esse aumento na eliminação, até o momento em que 
Tabela 4 - Valores médios e erro padrão da média (SEM) de Escherichia coli contado em $\log _{10}$ unidades formadoras de colônia por grama de fezes de leitões alimentados com dietas contendo probiótico e desafiados com E. coli

\begin{tabular}{|c|c|c|c|c|}
\hline \multicolumn{2}{|c|}{ Tratamento } & \multirow{2}{*}{$\begin{array}{l}\text { E. coli } \\
35 \text { dias }\end{array}$} & \multirow{2}{*}{$\begin{array}{l}\text { E. coli } \\
42 \text { dias }\end{array}$} & \multirow{2}{*}{$\begin{array}{l}\text { E. coli } \\
49 \text { dias }\end{array}$} \\
\hline Probiótico & E. coli & & & \\
\hline+ & + & 6,09 & 6,36 & 7,00 \\
\hline+ & - & 6,82 & 7,18 & 7,36 \\
\hline- & + & 7,54 & 5,36 & 8,00 \\
\hline- & - & 7,73 & 6,91 & 7,91 \\
\hline \multicolumn{2}{|c|}{ SEM } & 0,20 & 0,21 & 0,19 \\
\hline \multicolumn{5}{|c|}{ Efeitos principais } \\
\hline \multicolumn{2}{|c|}{ + Probiótico } & 6,45 & 6,77 & 7,18 \\
\hline \multicolumn{2}{|c|}{ - Probiótico } & 7,64 & 6,14 & 7,95 \\
\hline \multicolumn{2}{|c|}{+ E. coli } & 6,82 & 5,86 & 7,50 \\
\hline \multicolumn{2}{|c|}{ - E. coli } & 7,27 & 7,04 & 7,64 \\
\hline \multicolumn{5}{|c|}{ Fonte de variação $(\mathrm{P})$} \\
\hline \multicolumn{2}{|c|}{ Probiótico } & 0,0026 & 0,0916 & 0,0485 \\
\hline \multicolumn{2}{|c|}{ E. coli } & 0,2235 & 0,0026 & 0,7214 \\
\hline \multicolumn{2}{|c|}{ Probiótico x E. coli } & 0,4625 & 0,3292 & 0,5529 \\
\hline
\end{tabular}

foi realizada esta análise, não influenciou o desempenho destes animais. Vale ressaltar que só foi possível observar as diferenças entre os tratamentos desafiados, com e sem probiótico, mesmo que numéricas, aos 63 dias de idade, período esse em que se evidenciou a adaptação dos animais frente ao desafio, através dos diferenciais já referenciados.

\section{Discussão}

$\mathrm{Na}$ análise do GDPTotal, não foi verificado efeito significativo de interação e dos fatores isolados. No entanto, esta variável manteve um padrão similar ao observado no GDP63, sendo os melhores ganhos constatados nos tratamentos probiótico sem e com desafio 662 e 633g/dia, respectivamente. Nos tratamentos sem probiótico sem e com desafio os ganhos foram de 627 e $615 \mathrm{~g} /$ dia, respectivamente, houve uma pequena diferença de $12 \mathrm{~g} /$ dia entre os mesmos. Essa diminuição entre os ganhos foi propiciada pelo maior GDP49, oriundo do menor peso aos 35 dias (10,59 $\mathrm{kg}$ ) e o maior peso aos 49 dias de idade $(19,73 \mathrm{~kg})$ para os animais do grupo sem probiótico com desafio, resultando, portanto, numa diferença entre esses tratamentos de 95g/dia a favor do sem probiótico com desafio. É difícil predizer a resposta dos animais quando submetidos a diferentes desafios, dada à complexidade do envolvimento de fatores externos, além da pressão de infecção do ambiente e a adaptação da microbiota do animal, a qual tem como função a proteção da mucosa, sendo, portanto, bastante variadas às respostas encontradas ${ }^{20}$.

No ganho subseqüente (GDP63) houve uma inversão no desempenho, pois o aumento foi maior para os leitões sem probiótico sem desafio (120g/dia). Os animais que foram apenas desafiados com E. coli apresentaram reflexo negativo no ganho de peso após 14 dias da inoculação, que repercutiu de forma mais branda no período total. Dentre os tratamentos desafiados pôde-se observar que o probiótico com desafio manteve um ganho diferenciado em relação ao sem probiótico com desafio (633g/dia vs 615g/dia, respectivamente), resultando em $18 \mathrm{~g} / \mathrm{dia}$ a favor do tratamento com probiótico.

Diversos resultados têm sido encontrados, na dependência de vários fatores ${ }^{21} \mathrm{e}$ dentre estes, o que merece destaque é a influência das condições sanitárias do ambiente, as quais representam, na realidade, um desafio natural para os leitões recém desmamados, aliada à própria condição do estabelecimento da microbiota. 
Desta maneira, os resultados revelaram-se interessantes principalmente nas condições em que foi desenvolvido. Na literatura, resultados semelhantes foram relatados por Wang et al..$^{22}$, nos quais verificaram melhora tanto no ganho de peso como na conversão alimentar em leitões que o receberam, comparado aos que não receberam Lactobacillus fermentum I5007 células $\left(10^{8} \mathrm{UFC} / \mathrm{mL}\right)$, por via oral.

Constatações semelhantes foram identificadas em outros estudos ${ }^{23}$, nos quais é ressaltada a ação do probiótico Lactobacillus acidophilus 1F221, principalmente promovendo seu efeito pelo mecanismo de exclusão competitiva, resultando na melhor integridade intestinal, maior absorção de nutrientes e melhor desempenho. Além disso, tem sido demonstrado que o L. fermentum tem alta adesão às células Caco-2 e à mucosa intestinal, favorecendo a exclusão competitiva contra patógenos ${ }^{24}$, o que pode resultar na melhora da integridade da mucosa entérica.

Em contrapartida, Collinder et al. ${ }^{25}$ não verificaram esta melhora no ganho de peso de leitões suplementados com 1x $10^{7}$ UFC de Bacillus licheniformes/ grama de ração.

Junqueira et al. ${ }^{26}$ pondera sobre a melhora em alguns índices de produção, enquanto Trujano e Arellano ${ }^{27}$ relatam a redução de episódios de diarréia causados por agentes diversos e ainda Avila et al..$^{28}$ destacam o potencial dos probióticos em substituir alguns antibióticos.

No presente estudo após 28 dias da inoculação, os leitões do tratamento sem probiótico com desafio vieram a apresentar o menor $(\mathrm{P}>0,05)$ peso $(27,82 \mathrm{~kg}) \mathrm{em}$ comparação aos demais tratamentos $(29,81,28,91$ e 28,57 kg para probiótico sem desafio, probiótico com desafio e sem probiótico sem desafio, respectivamente), demonstrando a ação da E. coli. Ao compararmos os animais dos tratamentos sem probiótico com desafio e probiótico com desafio verificamos um diferencial numérico de $1,09 \mathrm{~kg}$ a favor do probiótico com desafio. Esse diferencial mostra um valor adicional represen- tativo na produção animal. Já para os leitões que não receberam probiótico (com e sem desafio), os pesos foram inferiores aos demais tratamentos (27,82 e 28,57 vs 28,91 e 29,81, sem probiótico com e sem desafio vs probiótico com e sem desafio, respectivamente). Se avaliarmos o probiótico sem desafio em comparação ao sem probiótico sem desafio percebe-se que o probiótico colaborou no desenvolvimento dos leitões, pois os mesmos tiveram um acréscimo de $1,24 \mathrm{~kg}$ no peso aos 63 dias de idade. Cumpre ainda acrescentar que o diferencial é ainda maior a favor do probiótico $(1,99$ $\mathrm{kg}$ ), quando da comparação entre o probiótico sem desafio e sem probiótico com desafio: $(29,81 \mathrm{~kg}$ e 27,82 $\mathrm{kg}$, respectivamente). Portanto, em situações com ou sem desafio, o probiótico mostrou resposta positiva no peso ao término da fase de creche.

O emprego do desafio é comumente utilizado como modelo no estudo da diarréia pós-desmame ${ }^{15}$. No estudo, a E. coli provocou diarréia nos leitões desafiados, sugerindo que a cepa foi capaz de colonizar e proliferar no intestino delgado, causando uma diarréia leve com reflexos negativos menos acentuados no desempenho dos animais. Essa diarréia leve, segundo a classificação preconizada no estudo, não foi encontrada por Wang et al. ${ }^{22}$, em cujo estudo os autores citaram que, inicialmente, os leitões de ambos os grupos, controle e probiótico, pareciam saudáveis e com fezes normais.

O desafio programado ocorreu 14 dias pós-desmame, de modo aos animais se adaptarem às novas condições de hierarquia e ausência da mãe. Além desses fatores, resíduos alimentares não digeridos e não absorvidos poderiam servir como substratos para a microbiota intestinal, elevando a produção de ácido lático e de ácidos graxos de cadeia curta. Tais argumentações foram confirmadas por Etheridge, Seerley e Wyatt ${ }^{29}$, Nabuurs et al. ${ }^{30}$ e Utiyama et al. ${ }^{31}$.

Comparativamente ao estudo de Wang et al. ${ }^{22}$, em que os leitões vieram a apresentar diarréia leve em 12, 24 e 48 horas após o desafio com E. coli, a suple- 
mentação de L. fermentum I5007 não atenuou esses efeitos, tendo a ocorrência da diarréia diminuído significativamente somente após 10 dias. O presente estudo obteve tal significância a partir de 24 horas após o desafio.

Aos nove dias após o desafio o escore de fezes dos animais sem probiótico com desafio ainda se mantinha elevado, comparado aos demais tratamentos, mas de maneira semelhante ao resultado encontrado por Wang et al. ${ }^{22}$, houve uma redução no valor do escore, porém sem significância estatística.

Konstantinov et al. ${ }^{32}$ verificaram um aumento do número de dias com escore fecal maior que 2 , nos leitões alimentados com a dieta contendo $10^{10} \mathrm{UFC} / \mathrm{mL}$ de L. sobrius DSM 16698. Esse resultado não corrobora com os achados, uma vez que os leitões não apresentaram escore fecal maior que 1,67. Essa observação leva a uma interpretação não incisiva gerando, porém, evidências quanto à resposta positiva do probiótico.

Já Taras et al. ${ }^{33}$, constataram uma reduzida incidência de fezes líquidas dos 28 aos 56 dias, nos leitões que receberam o probiótico com Enterococcus faecium NCIMB 10415.

Em contrapartida, os estudos de Utiyama et al. ${ }^{31}$ concluíram que os tratamentos empregados (antimicrobiano; probiótico: 1300 ppm à base de B. subtilis e $B$. licheniformis $\left(4,16 \times 10^{9}\right.$ de esporos viáveis $/ \mathrm{kg}$ de ração); prebiótico e extrato vegetal) não foram eficientes para controlar a diarréia no período pós-desmame.

Esse aumento na população de coliformes pós-desmame tem sido relatado como um fato normal ${ }^{34}$, uma vez que estudos prévios realizados por Konstantinov et al. ${ }^{32}$ demonstraram que as concentrações de E. coli aumentaram, em detrimento da redução do número de lactobacilos após o desmame de leitões. Contudo, tal aumento não foi observado nos grupos de leitões alimentados com dieta suplementada com antibiótico ou probióticos.

Do mesmo modo, Bogovic Matijasic et al. ${ }^{35}$ relataram aumento na contagem de coliformes em leitões do grupo controle (sem probiótico) em comparação aos que receberam probiótico (Lactobacillus gasseri K7 e LF221). Embora esses pesquisadores não tenham observado a mesma tendência em suínos alimentados com dietas suplementadas com os probióticos, as diferenças foram atribuídas mais às variações entre os animais, do que ao aditivo. Outros estudos têm demonstrado que a administração de probióticos causa uma redução na contagem dos coliformes nas fezes dos leitões ${ }^{36}$, talvez por um mecanismo de exclusão competitiva $^{37}$.

Vale ressaltar que, aos 35 dias de idade, verificamos que os leitões do tratamento sem probiótico com desafio apresentaram a segunda maior quantidade de $E$. coli nas fezes (7,54 $\log _{10}$ UFC/g fezes), associada ao maior escore fecal obtido 24 horas após o desafio, variando entre diarréia leve a moderada, o que pode ter repercutido no menor peso alcançado em comparação aos demais tratamentos. Já aos 42 dias houve uma queda na eliminação da $E$. coli nas fezes $\left(5,36 \log _{10}\right.$ $\mathrm{UFC} / \mathrm{g}$ fezes) as quais ainda estavam numa situação de diarréia leve a moderada, embora tenha ocorrido uma redução no escore fecal de 1,67 para 1,50.

Uma possível explicação para este fato seria que, nos animais do tratamento sem probiótico com desafio, a E. coli inoculada por via oral teria se aderido à mucosa intestinal com maior facilidade do que no sem probiótico sem desafio, uma vez que este último tratamento não recebeu E. coli LSS 164/04 diretamente. Em comparação aos tratamentos que já vinham recebendo o probiótico via ração, a bactéria também pode ter colonizado a mucosa, porém com maior dificuldade devido à presença das bactérias benéficas presentes no probiótico.

Partindo do princípio de que houve maior aderência de E. coli LSS 164/04 na mucosa dos animais do tratamento sem probiótico com desafio, aos 42 dias verificamos uma menor $(\mathrm{P}>0,05)$ quantidade desta bactéria nas fezes, comparativamente aos demais tratamentos $\left(5,36\right.$ vs 6,$36 ; 7,18$ e $6,91 \log _{10}$ UFC/g fezes, 
sem probiótico com desafio vs probiótico com desafio, probiótico sem desafio e sem probiótico sem desafio, respectivamente). O que, de certa forma, se alinha com as observações de Konstantinov et al. ${ }^{32}$, que demonstraram aumento nas concentrações de E. coli em detrimento da redução do número de lactobacilos, após o desmame de leitões. Esse questionamento ainda carece de maiores detalhes, justamente relacionados ao nível de aderência, sendo necessárias maiores investigações a respeito.

\section{Referências}

1. BOMBA, A.; JONECOVA, Z.; KOSCOVA, J.; NEMCOVA, R.; GANCARCIKOVA, S.; MUDRONOVA, D.; SCIRANKOVA, L.; BULECA, V.; LAZAR, G.; POSIVAK, J.; KASTEL, R.; MAREKOVA, M. The improvement of probiotics efficacy by synergistically acting components of natural origin: A review. Biologia, v. 61, n. 6, p. 729-734, 2006.

2. MAZEL, D.; DAVIES, J. Antibiotic resistance in microbes. Cellular and Molecular Life Sciences, v. 56, n. 9/10, p. 742754, 1999.

3.ZANI, J. L.; DA CRUZ, F. W.; DOS SANTOS, A. F.; GILTURNES, C. Effect of probiotic Cenbiot on the control of diarrhea and feed efficiency in pigs. Journal of Applied Microbiology, v. 84, n. 1, p. 68-71, 1988.

4.DE ANGELIS, M.; SIRAGUSA, S.; BERLOCO, M.; CAPUTO, L.; SETTANNI, L.; ALFONSI, G.; AMERIO, M.; GRANDI, A.; RAGNI, A.; GOBBETTI, M. Selection of potential probiotic lactobacilli from pig feces to be used as additives in pelleted feeding. Research in Microbiology, v. 157, n. 8, p. 792-801, 2006.

5.BUDINO, F. E. L.; THOMAZ, M. C.; KRONKA, R. N.; NAKAGHI, L. S. O.; TUCCI, F. M.; FRAGA, A. L.; SCANDOLERA, A. J.; HUAYNATE, R. A. R. Effect of probiotic and prebiotic inclusion in weaned piglet diets on structure and ultra-structure of small intestine. Brazilian Archives of Biology and Technology, v. 48, n. 6, p. 921-929, 2005.

6. ALNO, J. P.; NORMAND, V.; DELFORGE, J. Impact of a probiotic yeast Saccharomyces cerevisae boulardii on Clostridium difficile neonatal diarrhea in piglets. In: INTERNATIONAL PIG VETERINARY SOCIETY, 18., 2004, Hamburg. Proceedings... Hamburg: IPVS, 2004. p. 723.

7. MENTEN, J. F. M. Aditivos alternativos na produção de aves: probióticos e prebióticos. In: REUNIÂO ANUAL DA SOCIEDADE BRASILEIRA DE ZOOTECNIA, 2001, Piracicaba. Anais... Piracicaba: FEALQ, 2001. p. 141-157.

8.BOMBA, A.; NEMCOVÁ, R.; GANCARRCIKOVA, S.; HERICH, R.; PISTL, J.; REVAJOVA, V.; JONECOVA, Z.; POSIVAK, J. The influence of omega- 3 polyunsaturated fatty acids (Omega-3 pufa) on lactobacilli adhesion to the intestinal mucosa and on immunity in gnotobiotic piglets. Berliner und Munchener Tierarztliche Wochenschrift, v. 116, n. 7/8, p. 312-316, 2003.

9. LEEDLE, J. Probiotics and DFM's: mode of action in the gastrointestinal tract. In: SIMPÓSIO SOBRE ADITIVOS ALTERNATIVOS NA NUTRIÇÃO ANIMAL, 2000,

\section{Conclusão}

Concluiu-se que, em condições de desafio programado, o probiótico atua favoravelmente na redução da Escherichia coli e da diarréia, evidenciando resposta positiva em relação ao desempenho dos leitões, manifestado principalmente no peso médio ao final da creche.

Agradecimento - Os autores gostariam de agradecer a Novartis Saúde Animal Ltda pelo auxílio financeiro para a realização desse projeto.

Campinas. Anais... Campinas: Colégio Brasileiro de Nutrição Animal, 2000. p. 25-40.

10.JIN, L. Z.; HO, Y. W.; ABDULLAH, N.; JALALUDIN, S. Probiotics in poultry: modes of action. World's Poultry Science Journal, v. 53, n. 4, p. 351-368, 1997.

11.ALEXOPOULOS, C.; GEORGOULAKIS, I. E.; TZIVARA, A.; KYRIAKIS, C. S.; GOVARIS, A.; KYRIAKIS, S. C. Field evaluation of the effect of probiotic-containing Bacillus licheniformis and Bacillus subtilis spores on the health status, performance, and carcass quality of grower and finisher pigs. Journal of Veterinary Medicine Serie A, Physiology, Pathology, Clinical Medicine, v. 51, n. 6, p. 306-312, 2004.

12.KIERS, J. L.; MEIJER, J. C.; NOUT, M. J.; ROMBOUTS, F. M.; NABUURS, M. J.; VAN DER MEULEN, V. D. Effect of fermented soya beans on diarrhea and feed efficiency in weaned piglets. Journal of Applied Microbiology, v. 95, n. 3, p. $545-552,2003$.

13.POLLMANN, D. S.; DANIELSON, D. M.; WREN, W. B.; PEO JR, E. R.; SHAHANI, K. M. Influence of Lactobacillus acidophilus inoculums on gnotobiotic and conventional pigs. Journal of Animal Science, v. 51, n. 3, p. 629-637, 1980.

14.ISOLAURI, E.; SUTAS, Y.; KANKAANPAA, P.; ARVILOMMI, H.; SALMINEN, S. Probiotics: effects on immunity. The American Journal of Clinical Nutrition, v. 73, p. 444S-450S, 2001. Supplement, 2.

15.VAN DIJK, A. J.; ENTHOVEN, P. M.; VAN DEN HOVEN, S. G.; VAN LAARHOVEN, M. M.; NIEWOLD, T. A.; NABUURS, M. J.; BEYNEN, A. C. The effect of dietary spray-dried porcine plasma on clinical response in weaned piglets challenged with a pathogenic Escherichia coli. Veterinay Microbiology, v. 84, n. 3, p. 207-218, 2002.

16.TURNER, J. L.; DRITZ, S. S.; HIGGINS, J. J.; HERKELMAN, K. L.; MINTON, J. E. Effects of a Quillaja saponaria extract on growth performance and immune function of weaning pigs challenged with Salmonella typhimurium. Journal of Animal Science, v. 80, n. 7, p. 1939-1946, 2002.

17. BRUNO, D. G. Efeito de um fito compost no desempenho de leitões submetidos ao desafio experimental com Salmonella typhimurium. 2008. 137 f. Dissertação - Faculdade de Medicina Veterinária e Zootecnia, Universidade de São Paulo, Pirassununga, 2008.

18. NATIONAL RESEARCH COUNCIL (NRC). Nutrients Requirements of Swine. $10^{\text {th }}$ revised edition. Washington, D.C.: National Academy of Sciences, 1998. 189 p. 
19. STATISTICAL ANALYSIS SYSTEM (SAS). SAS User's guide: statistical analysis system. release 6.12. Cary: SAS Institute, 1998.

20.ANADON, A. A.; MARTÍNEZ-LARRAÑAGA, M. R.; MARTÍNEZ, M. A. Probiotics for animal nutrition in the European Union. Regulation and safety assessment. Regulatory Toxicology and Pharmacology, v. 45, n. 1, p. 9195, 2006.

21.MILES, R. D. Manipulation of the micro flora of the gastrointestinal tract: natural ways to prevent colonization by pathogens. In: ALLTECH'S NINTH ANNUAL SYMPOSIUM, 1993, Nicholasville. Proceedings... Nicholasville: Alltech Technical, 1993. p. 133-150.

22.WANG, A.; YU, H.; GAO, X.; LI, X.; QIAO, S. Influence of Lactobacillus fermentum $\mathrm{I} 5007$ on the intestinal and systemic immune responses of healthy and $E$. coli challenged piglets. Antonie Van Leeuwenhoek, v. 96, n. 1, p. 89-98, 2009.

23.ROGELJ, I.; BOGOVIC MATIJASIC, B.; CANZEK MAJHENIC, A.; STOJKOVIC, S. The survival and persistence of Lactobacillus acidophilus 1F221 in different ecosystems. International Journal of Food Microbiology, v. 76, n. 1/2, p. 83-91, 2002.

24.Li, J.; LI, D.; GONG, L.; MA, Y.; HE, Y.; ZHAI, H. Effects of live yeast on the performance, nutrient digestibility, gastrointestinal microbiota and concentration of volatile fatty acids in weanling pigs. Archives of Animal Nutrition, v. 60, n. 4, p. $277-288,2006$

25. COLLINDER, E.; CARDONA, M. E.; BERGE, G. N.; NORIN, E.; STERN, S.; MIDTVEDT, T. Influence of zinc bacitracin and Bacillus licheniformis on microbial intestinal functions in weaned piglets. Veterinary Research Communications, v. 27, n. 7, p. 513-526, 2003

26.JUNQUEIRA, O. M.; RODRIGUES, E. A.; BARBALHO, R. L. C.; PORTELA, G.; MORETTI, A. S.; LOPES, E. L. O. O efeito do uso de antibióticos ou probióticos em rações sobre o desempenho de suínos na fase inicial do crescimento. In: CONGRESSO BRASILEIRO DA ABRAVES, 10., 2001, Porto Alegre. Anais... Porto Alegre: ABRAVES, 2001. p. 317-318.

27. TRUJANO, M.; ARELLANO, P. Use of Saccharomyces cerevisae to control Isospora suis in piglets. In: IPVS CONGRESS, 18., 2004, Hamburg. Proceedings... Hamburg: International Pig Veterinary Society, 2004. p. 729.

28. AVILA, F. A.; SCHOKEN-ITURRINO, R. P.; QUINTANA, J. L.; BASSO, A.; AMBROSIM, J. A. Utilização de vacina e probiótico no controle de diarréia enterotoxigência em suínos causada pela Escherichia coli. Arquivos Brasileiros de Medicina Veterinária e Zootecnia, v. 50, n. 5, p. 505-511, 1988.
29.ETHERIDGE, R. D.; SEERLEY, R. W.; WYATT, R. D. The effects of diet on performance, digestibility, blood composition and intestinal micro flora of weaned pigs. Journal of Animal Science, v. 58, n. 6, p. 1396-1402, 1984.

30.NABUURS, M. J.; HOOGENDOORN, A.; VAN DER MOLEN, E. J.; VAN OSTA, A. L. Villus height and crypt depth in weaned and unweaned pigs, reared under various circumstances in the Netherlands. Research in Veterinary Science, v. 55, n. 1, p. 78-84, 1993.

31.UTIYAMA, C. E.; OETTING, L. L.; GIANI, P. A.; RUIZ, U. S.; MIYADA, V. S. Efeitos de antimicrobianos, prebióticos, probióticos e extratos vegetais sobre a microbiota intestinal a freqüência de diarréia e o desempenho de leitões recémdesmamados. Revista Brasileira de Zootecnia, v. 35, n. 6, p. 2359-2367, 2006.

32.KONSTANTINOV, S. R.; SMIDT, H.; AKKERMANS, A. D.; CASINI, L.; TERVISI, P.; MAZZONI, M.; De FILIPPI, S.; BOSI, P.; VOS, W. M. Feeding of Lactobacillus sobrius reduces Escherichia coli F4 levels in the gut and promotes growth of infected piglets. FEEMS Microbiology Ecology, v. 66, n. 3, p. 599-607, 2008 .

33.TARAS, D.; VAHJEN, W.; MACHA, M.; SIMON, O. Performance, diahrrea incidence and occurrence of Escherichia coli virulence genes during lon-term administration of a probiotic Enterococcus faecium strain to sows and piglets. Journal of Animal Science, v. 84, n. 3, p. 608-617, 2006.

34. MATHEW, A. G.; FRANKLIN, M. A.; UPCHURCH, W. G.; CHATTIN, S. E. Influence of weaning age on ileal micro flora and fermentation acids in young pigs. Nutrition Research, v. 16, n. 5, p. 817-827, 1996.

35.BOGOVIC MATIJASIC, B.; STOJKOVIC, S.; SALOBIR, J.; MALOVRH, S.; ROGELJ, I. Evaluation of the Lactobacillus gasseri $\mathrm{k} 7$ and LF221 strains in weaned piglets for their possible probiotic use and their detection in the feces. Animal Research, v. 53, n. 1, p. 35-44, 2004.

36.CHANG, Y. H.; KIM, J. K.; KIM, H. J.; KIM, W. Y.; KIM, Y. B.; PARK, Y. H. Selection of a potential probiotic Lactobacillus strain and subsequent in vivo studies, Antonie Van Leeuwenhoek, v. 80, n. 2, p. 193-199, 2001.

37.GARDINER, G. E.; CASEY, P. G.; CASEY, G.; LYNCH, P. B.; LAWLOR, P. G.; HILL, C.; FITZGERALD, G. F.; STANTON, C.; ROSS, R. P. Relative ability of orally administered Lactobacillus murinus to predominate and persist in the porcine gastrointestinal tract. Applied and Environmental Microbiology, v. 70, n. 4, p. 1895-1906, 2004. 